\title{
Arroganz und Verwundbarkeit
}

Edgar Wolter

"Vulnerabilität: eine, wörtlich,Verletzbarkeit, die zwar erst einmal nur die Anfälligkeit für psychische Krankheiten meint, aber durchaus auch als Dünnhäutigkeit zu lesen ist, als eine Art überempfindliche

Rezeptivität, welche die Alltagswelt schnell zur Überforderung werden lässt. Zu viele Wahrnehmungen, zu viele Blicke, und die Denke des anderen wird stets miteinberechnet, so dass die Außenperspektive den Innenblick dominiert. « ${ }^{1}$

\section{Intro 1.}

Ich bin nicht Edgar Wolter (allerdings kenne ich einen).

\section{Intro 2.}

These T: Über $x$ kann nur schreiben, wer $x$-förmig ist. Beispiele:

(i) Über Kunst kann nur schreiben, wer ... ja, was müsste man nun sagen?: ... wer Künstler ist? Oder: ... wer künstlerisch interessiert ist? Oder: ... wer ästhetisch und ggf. kunsthistorisch gebildet ist?

(ii) Zum religiösen Glauben vermag sich allein diejenige $\mathrm{zu}$ äussern, die selbst an einem solchen Glauben partizipiert (was auch immer das heissen mag). ${ }^{2}$

(iii) Über Arroganz können nur Arrogante schreiben.

(iv) Zu Verletzungen wird nur jemand etwas sagen können, der selbst einmal verletzt worden ist.

\footnotetext{
1 Thomas Melle, Die Welt im Rücken, Berlin 2016, 17 - Thomas wird übermorgen den Deutschen Buchpreis erhalten; ich lasse das so stehen, selbst wenn ich mich irren sollte. Es ist Sonnabend (an der Ostsee sagt man nie "Samstag") der 15. Oktober, 13:01; offenbar hat es geregnet, was aber nur an den Reifen zu vernehmen ist, die über den Asphalt rauschen.

2 Gern würde ich hier eine Fussnote einfügen, die die (In)Konsequenzen dieser These für die Theologie ausbuchstabierte - aber ich kenne mich darin leider gar nicht aus; mein Feld ist und bleibt ja die Kybernetik. (- stimmt also (ii)?) Und Messdiener zu sein (man vergleiche die biographische Notiz am Ende), steht der Regelungstechnik`näher als der Gotteslehre.
} 
Nehmen wir das vorletzte Exemplar. Gemäss (iii) sieht es nicht gut für mich aus. Es bieten sich nur drei Möglichkeiten: Entweder bin ich nicht arrogant, wodurch mir die Lizenz zum Thema entzogen ist; oder ich kann über das besagte Thema schreiben (was nicht heisst, dass der Text deshalb gut wird), gerade weil ich arrogant bin; oder ich bin arrogant und schreibe über Arroganz, und der Text wird gut oder schlecht.

Korrektur: Die dritte Möglichkeit ist keine eigene Option, sondern bereits in der zweiten enthalten. Es war ein Trick, eine Finte - die ich selbst offenlege, aber erst nachdem sie die meisten nicht bemerkt haben werden. War das eine arrogante Geste Ihnen, den noch geneigten Lesern, gegenüber? Und war es arroganter, dieses Versteckspiel zu inszenieren? Und noch arroganter, es offenzulegen? Und am arrogantesten, Sie danach zu fragen? Vielleicht werden Sie schon etwas ungehalten, gar wütend auf mich sein. Ist es noch ein grosser Schritt, den Spiess - eine proto-militärische Metapher intentionaler Verwundung! - umdrehen zu wollen?

Aber so weit sind wir wohl noch nicht - zurück zum Thema und These T: Ausgeschlossen ist, zu meinem Leidwesen, die sympathischste Option: einen guten Text über Arroganz zu schreiben, ohne arrogant zu sein.

Ist man arrogant (im Sinne von hybrid, anmassend), wenn man $T$ und damit (iii) widerlegen möchte?

\section{Intro 3.}

Folgende Regeln gelten für die Lektüre des vorliegenden Textes:

Nummer 1: Unterlassen Sie ...

Nummer 2: Kommen Sie nicht auf die Idee, den Text zu ...

Nummer 3:Verboten sei, dass man ...

Habe ich mich sklar und deutlich ${ }^{3}$ ausgedrückt? (Kontrollfrage $F$ )

Lieber Leser, wie empfinden Sie einen solchen Umgang mit Ihnen? Jemand schreibt Ihnen vor, wie Sie mit dem Text umzugehen haben (was ja seinerseits nur wieder ein Text ist ... progressus ad infinitum!), dazu noch in einem nicht sehr einladenden Ton (bitte setzen Sie für >... entsprechende Anweisungen ein - Sie sehen, schon wieder eine Anweisung, aber doch immerhin etwas freundlicher). Also, nochmal: Sind Nummer $1-3 \& F$ ein arroganter Sprechakt? (Der noch dazu

3 Cf. René Descartes, Meditationes de Prima Philosophia. Lateinisch/Deutsch, Stuttgart 1999,Vierte Meditation, Abschnitt 17, 157: clare \& distincte. 
vollkommen un- und kontraproduktiv ist) $)^{4}$. Vielleicht werden Sie abwinken bei gleichzeitigem Schulterzucken, mich also kaum mehr ernst nehmen wollen? Aber das würde mich sehr stören, in einigen Fällen gar verletzen (aber nur in einigen).

\section{Intro 4.}

\section{Was hat das alles mit Verwundbarkeit zu tun?}

Schauen Sie bitten nochmals auf die Liste der vier obigen Beispiele und auf deren zweite Hälfte; da hiess es:

(iii) Über Arroganz können nur Arrogante schreiben.

(iv) $\mathrm{Zu}$ Verletzungen wird nur jemand etwas sagen können, der selbst einmal verletzt worden ist.

Lassen Sie sich nicht täuschen: Aus Beispielen werden meist keine Thesen extrapoliert, sondern für längst bevorzugte Thesen werden passende Beispiele gesucht. So auch hier, meine Vermutung lautet schlicht: (iii) und (iv) unterhalten eine klärungsbedürftige Liaison. Und es geht noch weiter: Bemüht man sich um diese Klärung, sammelt man Argumente für eine rehabilitierte Arroganz und Argumente gegen die Vorschussplausibilität, welche die Verletzbarkeit geniesst. Ich befürchte, ich werde mich mit diesen Erwägungen nicht beliebt machen. Wäre es arrogant zu sagen, dass einem das egal sein kann? Und wäre es schon anbiedernd, wenn es einem ganz und gar nicht egal bliebe?

\section{Teil 1. Arroganz}

Arroganz scheint eine Form der individuellen, manchmal kollektiven Existenz zu sein, in der eine Person, Gruppe oder Nation in bestimmter Weise über ihre Verhältnisse lebt. Alles kommt auf das richtige Verständnis von sin bestimmter Weise an. Nehmen wir eine konkrete Person, nennen wir sie ... Manfred. Manfred muss, um arrogant sein zu können, eine Eigenschaft haben, die als (im weiten Sinn) gut gilt, mit der aber ungut umgegangen wird, und zwar von ihm selbst. Konkret: Manfred kann nicht arrogant sein - und d.h. nichts anderes als: auf eine Audienz arrogant wirken; s.u. -, wenn

4 Verbote provozieren nicht selten ihre eigene Übertretung - nicht umgekehrt; dazu Slavoj Žižek, Die Puppe und der Zwerg. Das Christentum zwischen Perversion und Subversion. Aus dem Englischen von Nikolaus G. Schneider, Frankfurt a.M. 2003, 39, 114. 
er nicht etwas hat oder kann, das allgemein als solches anerkannt und wertgeschätzt wird: ökonomischen Erfolg (Fairness vorausgesetzt - schon ein Widerspruch?); ein herausragender Schachspieler sein (wenn der Nerd-Faktor nicht allzu hoch ist - schon ein Widerspruch?); faszinierende Ideen haben (die nicht geklaut sind); begeisterungsfähig für andere sein, ohne ... Also: Manfred verfügt, um arrogant sein zu können, über einen selbst kreierten Besitz, ein Vermögen im Sinne einer Fertigkeit oder über eine Charaktereigenschaft (welche kompatibel mit Arroganz ist; nochmals s.u.) - und: Er geht damit in einer als ganz unangemessen empfundenen, nämlich überheblichen Weise um.

Einwand: Es stimme nicht, dass Arroganz eine besondere Auszeichnung impliziere, zumal Durchschnittsbürger ebenso arrogant sein könnten. Genau gegen diese Vermutung richtet sich der Zusammenhang von Arroganz und einem Gut (Besitz, Vermögen, Charakter). Und der Grund: Wer über nichts verfügt (also z.B.: selbst erworbenen Besitz, eine aussergewöhnliche Fertigkeit, einen arroganzkompatiblen und dennoch bemerkenswerten Charakterzug) kann deshalb nicht arrogant sein, weil die Überheblichkeit dieses NichtManfred schlicht lächerlich wirken muss. Mein Eindruck ist also, dass sich arrogantes Auftreten nicht (per se) der Lächerlichkeit preisgibt, sodass Arroganz einen distinkten Effekt in sich trägt, während der eigentlich unauffällige Hochstapler ein anderes Register an »reactive attitudes $\aleph^{5}$ aktiviert.

Einwand: Diese Antwort sei zu pauschal, weil wir oft nicht wüssten, ob jemand dieses bestimmte Gut $X$ habe; die hier angeblich etablierte Differenz schmilzt also wieder zusammen. Antwort: Diese Beschreibung ist zu löchrig, um einen Einwand zu formulieren; denn: Wenn Manfred arrogant wirkt (zum Beispiel mit etwas angibt oder sich für überlegen usw. hält), wird das gerade in Abrede gestellte $X$ latent oder explizit vorausgesetzt (egal, ob es faktisch zutrifft oder nicht). Man stelle sich dazu unsere Reaktion auf die Enthüllung, wie es um dieses $X$ tatsächlich steht, vor - und hier gibt es nur zwei distinkte Fälle: Nicht-Manfred hat dieses $X$ nicht - und dann wirkt er nicht mehr arrogant (wie vielleicht zuvor), sondern lächerlich bis hin zu dem möglichen Fall, dass er einem leid tut, warum er es nötig habe, sich so zu präsentieren. Oder aber, ihm kommt $X$ tatsächlich zu, und nur dann ist die Möglichkeit erklärlich, dass wir im Verweis auf $X$ seine Arroganz vielleicht sogar entschuldigen, über sie hinwegsehen oder in manchen Fällen gar verstehen. Aber das

\footnotetext{
5 Peter Strawson, Freedom and Resentment, in: Proceedings of the British Academy 48 (1962), 1-25.
} 
können wir nur, wenn wir $X$ (ggf. fälschlicherweise) voraussetzen oder $X$ in der Tat gegeben ist. ${ }^{6}$ Arroganz und Lächerlichkeit zu unterscheiden, bleibt dennoch gewagt. Und:Von ihrer Unangemessenheit nimmt das nichts.

Noch ein Einwand: Was soll dieses implizite Lob der Arroganz? Arroganz wird hier als etwas umschrieben, das auf jeden Fall ein Gut enthält, sodass sich das Etikett des Arroganten in eine latente Wertschätzung wandelt, denn mit dem angeblichen Vorwurf wäre sofort das Eingeständnis verbunden, dass diese Person aber nur arrogant sein könne, weil sie etwas Wünschenswertes kann, hat oder ist. Antwort: Stimmt! Aber zwei weitere Abers: Aber 1: Ist der Einwand wirklich einer? ${ }^{7}$ Aber 2: Pseudo-Arroganz ohne dieses Gut mündet eben in Lächerlichkeit - das ist schlimm genug!)

Bislang ging es lediglich um eine umstrittene, aber aus meiner Sicht notwendige Bedingung der Möglichkeit, arrogant zu sein - nicht um Arroganz selbst. Gesagt aber wurde zumindest, dass Arroganz ein unangemessener Modus ist, mit dem skizzierten Gut $X$ umzugehen. Nicht der Besitz von $X$ ist demnach das Problem, sondern der Kern der Arroganz liegt in einem verfehlten Vollzug, sich zu $X$ zu verhalten. Und für dieses Fehlverhalten gibt es, so scheint es oder mir, zwei unterschiedliche Versionen, die man sich am besten verdeutlichen kann, indem die jeweilige Vermeidung der Arroganz beschrieben wird.

Der eine Fall könnte das aristotelische Modell genannt werden. Demnach gleicht der rechte Umgang mit $X$ der Auffindung einer Mitte zwischen zwei Extremen. Man erinnere sich an Aristoteles' Idee, eine Tugend bestünde darin, die mesotes zwischen zwei Lastern zu bezeichnen. Die Tugend der Tapferkeit sei zum Beispiel so zu lokalisieren, dass sie den Mittelwert auf einer Skala bezeichnet, deren Endpunkte die Untugenden (= Laster?) der Feigheit einerseits und andererseits dessen, was er `Tollkühnheit nannte, bilden. ${ }^{8}$ Der rechte Umgang mit $X$ müsste demnach im Mittel zweier Pole liegen, von

\footnotetext{
6 Zugegeben sei, dass es Mischformen gibt, sodass man lächerlich und arrogant zugleich sein kann; Lächerlichkeit und Arroganz beziehen sich dann auf unterschiedliche Referenzpunkte. Ein Beispiel: Donald J. Trump - er ist beides, lächerlich und arrogant. Lächerlich aus fataler Unkenntnis bei ausgeprägtem Hybridcharakter mit aussenpolitischen Ambitionen; arrogant mit Blick auf seinen Reichtum, den er (wie hoch sein Vermögen auch ausfallt) tatsächlich hat, mit dem er aber hausieren geht das aber ist nicht lächerlich, sondern ein Akt der Arroganz.

7 Wir haben öfter Fälle, in denen Kritik Anerkennung beinhaltet, etwa wenn fehlendes Verantwortungsgefühl bemängelt wird, was das Eingeständnis enthält, dass sich dies an eine Person mit Macht, Einfluss, Entscheidungsgewalt richtet.

8 Dazu Christoph Halbig, Der Begriff der Tugend und die Grenzen der Tugendethik, Berlin 2013, 179-185.
} 
denen einer als der Ort der Arroganz angesehen wird, während der Kontra- und Mittelpunkt erst noch zu erschliessen wären. Wie wäre es mit `Unterwürfigkeit` und `kritischem Selbstbewusstsein oder 'falsche Bescheidenheit und `Offenheit? Naja, ich gebe zu, diese terminologischen Angebote stehen der Aristotelischen Treffsicherheit in einigem nach. Ich kann mich nur damit entschuldigen, dass die Vagheit der hiesigen Begriffe systembedingt ist. Arroganz müsste also als das Ungleichgewicht einer kommunikativen Tugend beschrieben werden, die in die tugend-averse Übertreibung abgleitet. Selbstbewusstsein ohne Selbstkritik wäre dann mit Arroganz gleichzusetzen.

Den anderen Fall möchte ich den ironischen nennen. Dieser folgt einer anderen Struktur als den tugendethischen Mittelbestimmungen (oder der moralisch gebotenen Mittelmässigkeit).Vielmehr geht es hier um eine Logik des Dementierens, Zurücknehmens, der Revision. Etwas wird also vorgebracht - und daraufhin sogleich kassiert, verzweideutigt, relativiert, ...

Ich sehe, lieber Leser, der Text entgleitet mir schon wieder. Eigentlich hatte ich vor, so weiterzumachen wie auf den ersten Seiten das war doch ein passabler Auftakt, oder nicht? Und nun geht es mit dieser analytisch übertriebenen Unterscheidungsfreude oder -wut weiter. Soll ich bekennen, dass ich mich dabei vom Eindruck der eigenen Arroganz nicht ganz freisprechen kann? Denn als Autor führe ich Sie durch zahlreiche Gedanken - einige sind tatsächlich von mir -, aber ich manipuliere auch die ganze Zeit, indem ich Dinge verschweige, übergehe, um auf andere gerade den Akzent zu setzen. Ich bestimme die Agenda, ohne dass Sie einschreiten könnten. Soll ich einen Blog mit Kommentarfunktion anbieten? Vielleicht sollten wir uns lieber unterhalten, ein persönliches Gespräch! Aber ich sag's Ihnen gleich: heute lieber nicht.

Allerdings erhält man die Selbstbezichtigung, arrogant zu sein, nicht gratis. Warum? Naja, ich habe ja nun einmal den Text, wie er bislang vorliegt, geschrieben - und einige Dinge zur Arroganz gesagt. Meine Selbstkritik oder, sagen wir eher (by the way, welches 'wir??), mein Unbehagen muss also zu den obigen Ausführungen passen. Das heisst, ich müsste über ein $X$ verfügen, mit dem ich nicht angemessen umgehe - entweder verfehle ich die kommunikativ tugendhafte Mitte oder ich revidiere meine Arroganz gerade nicht im Modus des Ironischen (wobei beim Dementieren ja stets etwas vom Dementierten übrigbleibt - eine Spur, eine Fährte, ein kleiner, vielleicht gewollter Rest) ${ }^{9}$.

9 Niemand komme auf die sagenhafte Idee, hier eine Derrida-Fussnote einzufügen. 
Gehen wir mal davon aus, auch mein Text enthielte diese latente Arroganz dem Leser gegenüber, der sich gegen den auktorialen Führungsstil nun einmal nicht wehren kann. Habe ich aber nicht gerade dadurch, dass ich den Gedankengang einfach irgendwo unterbrochen habe, offengelegt, dass mir einigermassen klar ist, was hier gerade vor sich geht? War das nicht eine Art von Dementi, Zurücknahme, Revision? Soll das also die Ironie sein, die die Gefahr der Arroganz dem Leser gegenüber dadurch unterbindet, dass sie sich selbst zum Thema macht? Was ist und war daran ironisch? Vielleicht ist Ironie nicht die einzige Form des Dementis, die Arroganz wahrhaft eindämmen könnte. Nachdenklichkeit könnte eine veritable Alternative sein - jenes Zögern, das eine sofortige Antwort zurückhält, ein Innehalten, das für einen Moment zurückgeht zum zuvor Gesagten und es sich etwas verwundert-distanziert von Neuem anschaut. Kann noch arrogant sein, wer nachdenklich ist? (Ja, es gibt Fälle - Heiner Müller? -, aber sie sind nicht die Regel).

Ich darf zusammenfassen, vorläufig: Arrogant zu sein und arrogant $z u$ wirken, ist ein und dasselbe. Einwand: Aber wir kennen doch alle die Differenz zwischen unserem Empfinden und der Reaktion der anderen! Einwand zum Einwand: Oh ja, diese Differenz kenne ich sehr gut, lieber Einwänder, aber: Arroganz ist ein forensischer (und also kommunikativer, s.o.) Begriff, sodass Arroganz keine intrinsische Eigenschaft von etwas sein kann, sondern nur - immerhin und leider - eine der Aneignung und Rezeption. Arrogant kann man nur sein, wenn es jemand gibt, der einen für arrogant hält. ${ }^{10}$ Und wenn es eine Kollision im Empfinden der Arroganz geben sollte, dann ist es keine zwischen Arrogant-Wirken und Arrogant-Sein, sondern zwischen Arrogant-Wirken, und Arrogant-Wirken ${ }_{2}$. Oder: In einer Robinson-Crusoe-Welt gab es solange keine Möglichkeit zur Arroganz, bis deren Potenz durch Freitag auf jene einsame Insel gelangte.

Entschuldige, lieber Leser, das war keine wirkliche Zusammenfassung dessen, was ich zur Arroganz gesagt hatte - eine Bilanz soll ja nie was Neues gegenüber dem zu Bilanzierenden enthalten. Das wurde mir einmal beigebracht. Darauf verzichte ich hier mit Vergnügen. Nun aber weiter, ich habe noch andere Termine!

\footnotetext{
${ }^{10}$ Man hüte sich vor der Umkehrung des Satzes bzw. der modal leicht verschobenen Paraphrasierung: "Arrogant ist man nur, wenn es jemand gibt, der einen für arrogant hält." Wäre dieser Satz wahr, ginge die Welt unter, sofort - weil dann allein der Blick des anderen über das Urteil des Arrogantseins entschiede. Doch er tut es nicht (oder aus anderen Gründen); also ist der Satz falsch - der oben aber ist trotzdem zutreffend.
} 


\section{Nächster Teil. Vulnerabilität!}

Wie überhaupt von Verwundbarkeit sprechen, schreiben? Erfordert sie nicht einen ganz anderen Ton als jenen, den die Arroganz als Thema abverlangt (aber vielleicht verlangt Arroganz gar keinen eigenen Ton?). Eine zartere, zögerndere Sprache. Eine, die nicht verletzt, sondern Raum gibt, die nicht vorschnell und meinungsfreudig, gar streitlustig verallgemeinerte Behauptungen vorträgt, sondern auf ihre zerbrechlichen Phänomene warten könnte. Kann es solch eine Sanftheit eigentlich geben?

Verwundbarkeit, Verletzbarkeit - einfach Synonyme? Verwundbarkeit scheint die verkörperlichte, körpernahe Version der Verletzbarkeit zu sein; und dennoch wird diese Art des embodiment sogleich dementiert, weil der Begriff metaphorisch aufgeladen ist: verwundet zu sein, fungiert gerade nicht als Zustand dessen, was die Disposition der Verwundbarkeit als Potenz in Aussicht stellt. Verwundbarkeit ist also kein primär körperliches Phänomen, sondern ein seelisches (das klingt recht dualistisch - aber lasst mir doch für einen Moment dieses Dual).Verwundbarkeit meint ja keineswegs, über die nicht selten negative Kapazität zu verfügen, Narben, Brüche, Schnitte, Kratzer, tiefe Wunden davon zu tragen - ausser man verwendet diese Liste ihrerseits als übertragene Redeweise. Und gerade dies macht $>$ Verwundbarkeit semantisch so stark: die Andeutung des Körperlichen, ja Fleischlichen und das sofortige Zurücknehmen eben jener Konnotation. Diese Bewegung von Angebot und $>$ Nachfrage ${ }^{11}$ ist sehr viel stärker ausgeprägt als im Fall der /Verletzbarkeit‘, die ohne den besagten Umweg zum Ausdruck bringt, von jemandem menschlich getroffen zu sein. >Vulnerabilität ‘ wiederum könnte beide Konzepte umfassen und die skizzierte Differenz im metaphorischen Arsenal unterlaufen.

Bevor es weitergeht, möchte ich bekennen, dass ich >Verwundbarkeit für ein sehr schönes (ich sage mit Bedacht nicht swunderschönes`) Wort halte. Es trägt genau jene Zagheit in und an sich, die nötig sein mag, um über das Phänomen zu sprechen. `Nachdenklichkeit‘ ist ein ähnliches Klangphänomen, das mitschwingen lässt, was es sinnvoll zu sagen vornimmt. Klingt Ihnen das alles zu pastoral, zu weinerlich? Dann gebe ich gern ein anderes Beispiel mit demselben Effekt: 'Scheisser. Semantik und Tonalität gehen hier eine überaus erfolgreiche Beziehung ein, eine "geheime Verabredung« sozusagen.

\footnotetext{
11 ,Nachfrage in Anführungsstrichen: weil es nicht um Kommerz geht, vielmehr um den Akt des Nachfragens aus Irritation und Interesse.
} 
Sogleich wäre zu bedenken, wer denn von welcher ,Verwundbarkeit spricht. Derjenige, der selbst verwundet ist? Kaum, denn niemand sagt: "Ich bin verwundbar". Oder doch? - und wenn, an wen richtet sich diese intime Szene? Wohl an jemanden, der niemals diese Verwundbarkeit herausfordern würde. Oder umgekehrt gerade an den einzigen, der es könnte; diejenige, die man liebt.

Und genau jetzt - was für ein Zufall (sic!) - schreibt dieser Mensch: "Steckt das Wunder eigentlich auch in der Verwundbarkeit? Schön wäre es jedenfalls! «12 (Gern würde ich mit >ja antworten).

Aber wenn /Verwundbarkeit ` ein Teil der Aussenperspektive auf andere wäre? Doch auch hier zögere ich; denn wie schon im Fall der Selbstbeschreibung kommt das Wort deskriptiv auch in diesem Kontext kaum vor. Im Gegensatz zu ıverletzt< oder >verletzbar ist , Verwundbarkeit kein Wort unserer Umgangssprache, weit eher ein Abstraktum, fast ein literarisches Kunstwort, eine "formale Anzeige « ${ }^{13}$ der Anthropologie (und der Tierethik) ${ }^{14}$.

Weiter geht's, lieber Leser, kommen wir nach neun Seiten endlich zum Thema, ich werde mich kurzfassen. Versprochen! Verwundbarkeit und Arroganz - welch ungleiches, wunderbares Paar! Beides sind Eigenschaften, aber während Verwundbarkeit eine Disposition ausgedrückt, die allen passionierten Lebewesen zukommt, bezeichnet Arroganz eine Charaktereigenschaft, die längst nicht alle besitzen, glücklicherweise. Daraus ergibt sich der verquere Fall, dass alle das Vermögen zu einer Eigenschaft $V$ haben, ohne dass irgendjemand faktisch $V$ haben müsste (oder ist), versus der Möglichkeit, dass wenige die Eigenschaft $A$ haben, ohne von einer allgemeinen Disposition zu $A$ sprechen zu müssen. Oder einfacher:Alle sind verwundbar, aber nicht notwendigerweise verwundet. Einige sind arrogant, ohne die Disposition zur Arroganz zu haben (wenn der Term >Disposition< nicht völlig unterhöhlt werden soll - oder wir erfinden jetzt das Wort sarrogantbar).

Verwundbarkeit markiert eine Schwäche, die sich sogleich dialektisch (hier ist dieses abgegriffene Wort tatsächlich mal angebracht) ins Gegenteilige wendet. Dann nämlich, wenn man die eigene Verwundbarkeit annimmt, vielleicht gar offen zulässt. Ein Zeichen

\footnotetext{
${ }^{12}$ Vollkommen unautorisierte whatsapp-Nachricht. Es ist 11:41 am 28. Oktober. Fast habe ich vergessen, dass ich ja eigentlich an der Ostsee sein sollte. Sie sehen, es ist etwas Zeit vergangen seit Fussnote 1.

${ }_{13}$ Damit sei bei Heidegger so etwas gemeint wie eine vorläufige Bezugnahme; dazu Hubert Dreyfus/Charles Taylor, Die Wiedergewinnung des Realismus. Aus dem Englischen von Joachim Schulte, Berlin 2016, $297 f$.

${ }^{14}$ Vgl. Stephen Mulhall, The Wounded Animal. J.M. Coetzee and the Difficulty of Reality in Literature and Philosophy, Princeton 2008.
} 
der Stärke? Und Verwundbarkeitsreduktion als eigentliche Schwachund Sollbruchstelle? Ein ähnliches Spiel lässt sich im vollen Ernst mit Arroganz durchführen. Die angeblich starke Geste der gewollten und inszenierten Unberührbarkeit - noli me tangere ${ }^{15}$ - verflacht sogleich, wenn die Möglichkeit der Schwäche - nicht unbedingt die Schwäche selbst - ausgeschlossen, verdeckt, dementiert wird. Die Potenz der Ohnmacht als wahre Stärke? - der Gestus unangreifbarer Stärke als eigentliche Schwachstelle? Wir schrammen hier nah an der viel gescholtenen Küchenpsychologie vorbei.

Das muss noch nicht heissen, dass es falsch ist, sich die Dinge so zurecht $\mathrm{zu}$ legen - aber vielleicht ist es etwas zu naheliegend, um mich nicht noch deutlicher auszudrücken. Ohne Frage, der Verwundbarkeit von Menschen kommt eine moralisch gebotene Vorschlussplausibilität zu. Arroganz hingegen liegt auf dem anderen Ende der normativ aufgeladenen Skala menschlicher Be- und Abwertungen. Damit scheinen die Dinge vollkommen klar verteilt zu sein, und will man beide Extreme nicht einfach nebeneinander stehen lassen, fügt man die Zusatzthese zu: Arroganz berührt, stört, verletzt, ja >verwundet $\iota$ den, an den sie adressiert ist.

Es ist nicht ganz ungefährlich, diese naheliegende, sich fast aufdrängende Sicht herauszufordern. Aber es ist nun einmal gar nicht klar, dass `Arroganz die Verwundbarkeit des anderen (über)strapaziert. Weil gar nicht per se feststeht, was als $V$ und als $A$ gelten darf. In den allermeisten Fällen ist beides eine Sache des interaktiven Aushandelns, d.h. die hier betrachteten Phänomene der Verwundbarkeit und Arroganz sind nicht nur nicht einfach >da ; sondern:Verwundbarkeit und Arroganz gehören in ein (holistisches?) Netzwerk von Vermögen, Haltungen und Anerkennungsmechanismen, die sich allein im Zusammen- und Wechselspiel definieren. Das bedeutet nichts anderes, als dass es ohne Verwundbarkeit (in recht weitem Sinn) keine Arroganz geben könnte, weil niemand von ihr ge- und betroffen wäre (nochmals Auftritt: R. Crusoe). Und ohne Arroganz (bzw. analoge Mechanismen kommunikativer Friedlosigkeit) gäbe es zwar die Potenz des Vulnerablen, aber ohne eine einzige Wunde (wenn dies denn überhaupt möglich ist) ${ }^{16}$.

Mit zwei Fällen haben wir es also zu tun, wenn es um das Zusammenspiel von Arroganz und Verwundbarkeit geht:

_ durch Arroganz vverwundet $z$ u werden;

${ }^{15}$ Bitte keine überflüssige Anmerkung an dieser Stelle zu J.-L. Nancy - welch ein Selbstwiderspruch, dass ich das in einer Fussnote sage, nicht wahr?

${ }^{16}$ Woraus sich sogleich die Frage ergibt, woher wir von einer Disposition zu $x$ wüssten, wenn $x$ niemals aktualisiert worden wäre. 
_ das (angebliche?) >Verwundetsein`, welches Arroganz als seine Ursache bezichtigt und dabei selbst zum Problem wird.

Gibt es nicht noch mehr Fälle? Vielleicht; ich übergehe sie im Folgenden. Der erste Fall ist der herkömmliche - ihn haben wir schon gestreift, und so werde ich mich kurz fassen. Der zweite ist der interessantere - ihn möchte ich etwas genauer betrachten.

Der Vorwurf der Arroganz ist intrinsisch kritisch. Es gibt demnach keinen Fall, in dem der Arroganz als solcher etwas Gutes abgewonnen werden könnte - "als solcher" meint hier den Akt des Arroganten selbst, nicht dessen (vielleicht gar positive) Effekte oder andere begleitende charakterliche Züge (die sympathischer als die Arroganz sein mögen). Nach den obigen Bestimmungen meint Arroganz entweder die falsche Balance zwischen zwei kommunikativen Lastern (eines dieser beiden Laster wäre die Arroganz selbst), das Verfehlen einer ausgewogenen Mitte; oder aber das Fehlen kritischer Kommentierung des Arroganten durch Ironie, durch die jene Arroganz partiell revidiert wird; oder aber das Ausbleiben dessen, was Arroganz nicht nur zurücknimmt und dementiert, sondern in einen neuen kommunikativen Kontext versetzt und so womöglich beendet: Nachdenklichkeit.

Arroganz regt auf, stört, stösst ab - aber verwundet sie? Ja, in einem Fall kann sie das tun, demjenigen nämlich, in welchem Arroganz absichtlich eingesetzt wird. Es gibt eine intentionale (d.h. als intentional erlebte) Arroganz, die verletzen, auch verwunden kann. Implizite Arroganz hingegen, die jemand hat, ohne auf jemanden konkret gerichtet zu sein, gehört eher ins Register der Störung und Abstossung. Die Weisen der Entschuldigung, der Rücknahme, auch des möglichen Verzeihens rufen daher ein ganz unterschiedliches Repertoire auf je nach dem, welchen der beiden Fälle man betrachtet.

Intermezzo 1: Arroganz als Versuch, nicht verletzt zu werden, die Vorwegnahme des vermuteten Angriffs als faktische Reaktion auf etwas, das noch gar nicht stattgefunden hat. Würde es leichter fallen, mit jemanden umzugehen, den man für arrogant hält, wenn man dessen Arroganz als Reaktion auf dieses inichts verstünde?

Intermezzo 2: ^Arroganz` (nicht Arroganz) als kommunikatives Angebot. Eine kleine Anekdote dazu: Es gab einen berühmten Philosophen, der an einer bayrischen Universität lehrte; und er galt (und gilt) als die Ausgeburt blinder Arroganz in weisheitsliebenden Zirkeln. Wenn ein Student eine Verständnisfrage stellte, kam als Replik lediglich: "Lesen Sie gefälligst die Seiten 5-7 meines Aufsatzes noch einmal und endlich aufmerksam." Und nun traf ich letztens einen seiner ehemaligen Assistenten, einen mittlerweile noch berühmteren 
Philosophen, der seinen Lehrer vehement verteidigte.Viele Szenen schilderte er, etwa wie liebevoll jener Mann mit den Kindern seines damaligen Assistenten umging, ihnen Flugzeuge bastelte und vieles mehr. Und schliesslich sagte er: "Die angebliche Arroganz von ... war ein Angebot, gefolgt von einem Warten auf möglichen Protest, einen besseren Einwand, gerade gespickt mit leichter Wut, die sich herausfordern lässt. Immer noch seltsam genug, aber ..."

Liesse sich der Fall vielleicht auch so erzählen, dass das Gefühl der Verletzung durch Arroganz übersieht, dass eine Offerte vorlag, vollkommen offen miteinander zu sein? Ich zögere jetzt auch ... ein Warten auf Protest, der natürlich nicht kommt (Sie könnten mir aber schreiben; ich empfange allerdings nur Briefe, handschriftlich).

Wir nähern uns dem zweiten Fall, Verwundbarkeit als Problem. Dazu enthält der Text, der schon wieder viel zu lang wird, bereits zwei Anmerkungen. Arrogant sein heisst, auf jemanden, den man mit Arroganz ggf. verletzt, verwundet hat, arrogant zu wirken. Arroganz ist eine kommunikative Eigenschaft, sie liegt nicht einfach vor, sondern ist eingebettet in einen Diskurs, der sie lokalisiert und individuiert. Arroganz und Verwundung gehören folglich zu einem gemeinsam geteilten Arrangement, in dem überhaupt erst ausgehandelt wird, was als arrogant und verletzend gelten könnte - sie bedingen sich demnach gegenseitig. Was arrogant ist - i.e. unbalanciert, nicht revidiert durch Ironie, undementiert durch Nachdenklichkeit -, bleibt also ein prekäres Geben und Nehmen zwischen Absender und Rezipient, die zudem ihre Rollen ständig tauschen, wechseln, antizipieren, dem anderen überlassen, zuweisen und wieder einfordern.

Der zweite Hinweis führt zurück an den Beginn dieses vollkommen klaren Textes - wirf, lieber Leser, noch einmal einen Blick auf's Motto: Demnach könne Vulnerabilität »als Dünnhäutigkeit (gelesen werden), als eine Art überempfindliche Rezeptivität «. Eine vor allem auf sich selbst gerichtete "Dauerreflexion" wäre das, die den Blick von aussen gegenüber der Eigenwahrnehmung stets privilegiert. Und dies behauptet jemand, der die Abgründe unseres Duals von Arroganz, die abstösst und verletzt, und Verwundbarkeit, die sich bis an den Rand der Existenz biographisch beglaubigt hat, erleben und nun geradezu erschreiben musste. ${ }^{17}$ Nichts wird vom Versagen des Arroganten und der Faktizität begangener Verletzungen zurückgenommen, wenn - Thomas Melle folgend - ebenso die Möglichkeit erwogen wird, dass sich die Verhältnisse umdrehen können.

\footnotetext{
${ }^{17}$ Verwiesen sei nochmals freundlich auf die obige These $T$ plus (iv). Also doch richtig?
} 
Der Arroganzvorwurf aus Verletzungsanfälligkeit wird dann selbst zur isolierenden Strategie, die von einer spezifischen Asymmetrie zwischen Arroganz und Verwundbarkeit lebt. Denn die Normativitäten scheinen durch die verwendeten Etiketten klar zugeordnet zu sein, und so wird umso effektiver verschleiert, dass die Wertigkeiten diametral zum initialen Vorwurf verteilt sein mögen. Der Vorwurf der Arroganz gliche dann einem diskursiven Argument, das die reale Situation vorschnell hinter sich lässt, um von der eigenen Anfälligkeit, die sich schon immer von irgendjemandem getroffen wähnt, absehen zu dürfen. `Dünnhäutigkeit - wieder eine Metapher des Verleiblichten - stünde nun für den Mangel an (Frustrations)Toleranz für ein Gegenüber, dessen Andersheit nicht nur aberkannt, sondern gezielt moralisch diskreditiert wird.

Die vorschnelle Übernahme einer sakrifiziellen Rolle, die sich sogleich betroffen, im schlechtesten Sinne gemeint, missverstanden, abgewertet, übergangen fühlt, schottet sich absichtlich oder latent vom möglichen Gespräch ab. Die Kaskade der Entrüstung kennen wir: in seinem Innersten sei jemand getroffen worden, seine Integrität sei angetastet, die religiösen Gefühle missachtet und mit den Füssen (der Karikatur) getreten worden. Sicher, all dies mag zuweilen zutreffen - gar bis zur Selbstevidenz, die tatsächlich keine Fragen mehr zulässt.

Doch die moralisch aufgeladene Referenz auf jene Beschädigungen kann sich auch ihrerseits ins Unrecht setzen, indem sie viel zu früh in Anspruch genommen wird oder gänzlich deplatziert wirken muss. Könnte man von inverser Arroganz sprechen? Auch hier haben wir es mit den vertrauten, lediglich kaschierten Mechanismen des Kontaktabbruchs bei simultanem Überlegenheitsgefühl zu tun - nur als Unterlegenheit auf Zeit präsentiert. Einigen klingt das vielleicht zu sehr nach Nietzsche - »Aufstand der kleinen Mucker« (irgendwo im Antichrist). Und in der Tat hat die Marginalie arroganter Bezugnahme - und es ist eine Marginalie - auf die eigene Verwundbarkeit ein Element des Ressentiments an sich.

Wo vielleicht selbst gegen das zitierte Motto Einspruch erhoben werden könnte, ist die lautlose Gleichsetzung von `Dünnhäutigkeit< (als Pendant zum »dicken Fell« gefährlicher Ignoranz) mit >Rezeptivität<, ob überempfindlich oder nicht. Das Verdikt, durch die Arroganz des anderen verletzt zu sein, könnte sich auch und gerade am Mangel der Empfänglichkeit für das Aussen entzünden. Nicht mehr die Hermeneutik bestimmte dann das Bemühen umeinander, sondern dieses wird durch eine Hermetik sub contrario geradezu konterkariert. 
Und all dies gilt, selbst wenn man in eine sensible Beschreibung der Lage jene berührenden, oft schockierenden Fälle einbezieht, in denen sich die Frage, ob jemand wirklich verletzt, verwundet wurde, nicht mehr stellen darf. Sie tatsächlich zu stellen, bliebe hier nicht nur das Zeugnis gänzlich fehlender Empathie, sondern auch einer Arroganz, die für das Leid anderer (aspekt)blind geworden ist.

Und dennoch: Nicht Verwundbarkeit als Disposition, wohl aber der aktuale Umgang mit ihr kann ebenso ein Diskursprodukt sein wie das, was wir für arrogant halten. Und der Anspruch, auf die eigeneVerwundbarkeit Bezug nehmen zu können, ist daher fast genauso sensibel und zuweilen prekär, wie es die Verteidigung der Arroganz als Angebot an den anderen ist. Wird das nicht wahrgenommen, ähnelt der nicht mehr reflexive, sondern reflexhafte Hinweis auf erlittene Wunden womöglich selbst einer arroganten Szene, weil sie den anderen als anderen nicht mehr sehen, hören, spüren will. Und auch hier ist gesagt, dass die Referenz auf jene Verwundung als arrogante Geste nur dann wirklich arrogant sein kann, wenn ein Gut - ein Besitz, eine Fertigkeit, ein Charakterzug - vorliegt, mit dem auf ganz ungute Weise umgegangen wird. Die Gefahr, sonst in die Lächerlichkeit abzugleiten, besteht demnach auch hier.

Der Hinweis, verwundet zu sein, als Form ultimativer Arroganz? Wenn man das >ultimativ $<$ wieder streicht, dann: ja! Und auch das ist leider nicht ausgeschlossen: die Referenz auf die eigene Vulnerabilität als im Kern arroganter, unbalancierter, ironie- und witzloser Akt im Mangel an Nachdenklichkeit und Empathie. Genau um diese Potenz derVerwundbarkeit ging es hier, am Rande.

\section{Near the end 1.}

Macht man sich durch Arroganz verwundbar? Und ist man arrogant, wenn man im sofortigen Verweis auf mögliche Wunden intolerant mit sich selbst ist? (Ich gebe zu, das alles erinnert an Frischs Fragebogen).

\section{Near the end 2.}

Arroganz und der Umgang mit Verwundbarkeit sind kybernetische und kybernethische Eigenschaften. Sie sind Elemente einer Lehre der reziproken Steuerung und Regelung (das ist mein Fachgebiet; bitte vergessen Sie das nicht; ich kenne mich da aus). Dies trifft, das 
will ich schnell noch hinzufügen, für den Diskurs selbst auch zu: Ich tippe, dass die meisten anderen Autor^inn ${ }^{\star}$ n in diesen Blättern die Verwundbarkeit mit Verweis auf Opferperspektiven, im Modus der Achtsamkeit, im Register der Seelsorge verteidigen, schützen, wahren werden (aber es ist nur eine Vermutung). Der Diskurs über Verwundbarkeit wäre seinerseits Verwundungen ausgesetzt. Ich wertschätze diese Apologie - gerade, indem ich den entgegengesetzten Weg gewählt habe.

\section{Near the end 3.}

These T: Über $x$ kann nur schreiben, wer $x$-förmig ist. Beispiele:

(iii) Über Arroganz können nur Arrogante schreiben.

(iv) $\mathrm{Zu}$ Verletzungen wird nur jemand etwas sagen können, der selbst einmal verletzt worden ist.

Eigentlich wollte ich $T$ widerlegen. Ich glaube, das ging ziemlich daneben.

\section{Near the end 4: über den Autor, hinaus.}

Mein Name ist, in der Tat, Edgar Wolter.

- Edgar Wolter wurde an der Idaho State University in Kybernetik promoviert und lebt seitdem als Messdiener zurückgezogen an der Ostsee. Zahlreiche Schriften liegen von inm im Privatdruck bzw. Selbstverlag vor. 